\title{
Differing Responses in Growth and Spontaneous Mutation to Antibiotic Resistance in Bacillus subtilis and Staphylococcus epidermidis Cells Exposed to Simulated Microgravity
}

\author{
Patricia Fajardo-Cavazos, Raed Narvel, and Wayne L. Nicholson
}

Department of Microbiology and Cell Science, University of Florida, Merritt Island, FL

\begin{abstract}
Bacteria of the genera Bacillus and Staphylococcus are frequent inhabitants of the International Space Station (ISS) and represent possible opportunistic pathogens. The effect of simulated microgravity on growth and the frequency of mutation to antibiotic resistance in the model surrogate organisms Bacillus subtilis (B. subtilis) and Staphylococcus epidermidis (S. epidermidis) were investigated. The test organisms were cultivated for six days in Rotating Wall Vessel (RWV) clinostats either in the vertical (simulated microgravity) or horizontal (1 $g$ control) orientation. Parameters measured were: optical densities (ODs); viable counts; frequencies of resistance to rifampicin (RFM); and frequencies of double resistance to RFM and
\end{abstract}

Key words: Antibiotic Resistance; Bacillus subtilis; Clinorotation; International Space Station; Microgravity; Microorganisms; Mutation; Rifampicin; Staphylococcus epidermidis; Trimethoprim

Correspondence to: Wayne L. Nicholson Space Life Sciences Laboratory 505 Odyssey Way, Room 201-B Exploration Park at Kennedy Space Center Merritt Island, FL 32953

Telephone: 321-261-3773

E-mail:WLN@ufl.edu trimethoprim (TMP). The results indicated that the response to simulated microgravity differed in the two microorganisms. Both $B$. subtilis and $S$. epidermidis grew to higher ODs and cell numbers in simulated microgravity. However, the frequencies of mutation, both to RFM resistance and double resistance to RFM and TMP, were observed to increase significantly in simulated microgravity-grown $B$. subtilis but not in $S$. epidermidis.

\section{INTRODUCTION}

Preparations are underway for longduration missions through interplanetary space to destinations such as the Moon, near-Earth asteroids, or Mars (International Space Exploration Coordination Group, 2013). For more than five decades, human spaceflight missions into Low Earth Orbit (LEO) have rendered a wealth of information about the challenges of space travel and their effects on human health. Future missions beyond LEO are more likely to expose astronauts to higher risks to their health and performance. Of particular importance is the documented dysregulation of astronauts' immune systems during long-term missions (Crucian et al., 2009; Guéguinou et al., 2009), combined with the reported enhanced virulence of some microorganisms exposed to the stresses of the spaceflight environment, especially microgravity (Klaus and Howard, 2006; Ott et al., 2012). These two phenomena 
acting together could lead to an increase in astronaut infections by opportunistic pathogens during extended missions (Klaus and Howard, 2006). Committees representing both the National Research Council (NRC) and the International Space Exploration Coordination Group (ISECG) have identified the need to better understand health risks during space exploration, and both organizations have recognized the ISS as the best available platform to conduct research activities to address these challenges (ISECG, 2013; National Research Council, 2014).

Although space stations start out as essentially sterile environments, microbes rapidly colonize numerous ecological niches, to which they adapt and evolve in response to selective pressures unique to the spaceflight environment (Novikova, 2004; van Tongeren et al., 2007). Although preflight protocols minimize the risk of astronaut infection by true pathogens, a number of opportunistic pathogens have been isolated from space station crew quarters and from their human inhabitants, including species of the genera Bacillus, Citrobacter, Enterobacter, Enterococcus, Escherichia, Flavobacterium, Haemophilus, Klebsiella, Morganella, Proteus, Pseudomonas, Ralstonia, Serratia, Staphylococcus, Stentrophomonas, Streptococcus, and Yersinia (Ilyin, 2005; Klaus and Howard, 2006). In fact, fungal infections, viral diseases, styes, and infections of the urinary tract, upper respiratory tract, and subcutaneous tissue all have been documented from STS-1 to STS-108 missions (Sams, 2009). In particular, Bacillus and Staphylococcus spp. are the most ubiquitous organisms from the ISS, especially from crew quarters, debris, and lint (Venkateswaran et al., 2014). Not surprisingly, S. epidermidis was the most frequently encountered organism in the ISS microbiome, due to its close association as a skin commensal of humans (Venkateswaran et al., 2014).

Knowledge about the efficacy of antimicrobial interventions to treat infections during spaceflight is limited given the difficulty to assess the interplay between pharmacokinetics and physiologies of host and microbiome, both of which are altered in the spaceflight environment (Wotring, 2012). Decreasing microbial antibiotic susceptibility in the microgravity environment has been demonstrated from experiments performed on Salyut 7 (Tixador et al., 1985) and the Space Shuttles Challenger (Lapchine et al., 1986) and Discovery (Tixador et al., 1994). In addition, experiments performed on space station Mir showed that the frequency of mutations to streptomycin resistance in the E. coli rpsL gene was increased by 2- to 3-fold in space compared to ground controls, and the spectrum of mutations observed was clearly altered (Fukuda et al., 2000; Yatagai et al., 2000). Experiments onboard the ISS have demonstrated that horizontal transfer of antibiotic resistance plasmids can occur among both Gram-positive and Gram-negative bacteria (De Boever et al., 2007). The above observations indicate that the possibility must be considered of antibiotic-resistant strains emerging, becoming dominant types in the microbiomes of crew members, and causing health problems.

Opportunistic infections are often treated with combinations of two antibiotics that differ in their mechanisms of action. Two antibiotics that were prescribed together to combat acute and recurrent infections are rifampicin (RFM) and trimethoprim (TMP) (Stein et al., 1988). Although the RFMTMP combination regimen is no longer recommended (Zander et al., 2010), they are still indicated as components of multidrug therapies (Centers for Disease Control and Prevention, 2011; Cosgrove and Avdic, 2013). In addition to its clinical relevance, RFM resistance studies have been invaluable in understanding cellular processes and global responses of bacteria to different environments (Maughan et al., 2006; Maughan et al., 2004; Nicholson and Maughan, 2002). RFM is a broad-spectrum antibiotic that inhibits bacterial transcription initiation (Wehrli et al., 1968) by binding to the $\beta$-subunit of RNA polymerase in the mRNA exit channel, 2-3 nucleotides downstream from the active site (Campbell et al., 2001). Bacterial resistance to $\mathrm{RFM}\left(\mathrm{RFM}^{\mathrm{R}}\right)$ results from mutations in the $r p o B$ gene encoding the $\beta$ subunit of RNA polymerase, particularly in a small area called Cluster I corresponding to the RFM binding site (Jin and Gross, 1988). By comparison, TMP is a folate analogue that exerts its antimicrobial activity by competitively inhibiting dihydrofolate reductase (DHFR), an enzyme that catalyzes the reduction of dihydrofolate to tetrahydrofolate. Resistance to TMP most commonly stems from a chromosomal mutation in the $d f r A$ gene that results in the 
production of a DHFR that binds less tightly to TMP (Gleckman et al., 1981). In S. aureus, TMP ${ }^{\mathrm{R}}$ is caused by mutations that change DHFR conserved amino acids L41F, F99Y/S, or H150R (Vickers et al., 2009). TMP ${ }^{\mathrm{R}}$ can also result from elevated expression or activity of DHFR or dihydropteridine synthase (DHPS). In addition, it has been reported that mutations in $r p o B$ causing $\mathrm{RFM}^{\mathrm{R}}$ can up-regulate expression of the DHFR gene $d f r A$, also resulting in $\mathrm{TMP}^{\mathrm{R}}$ (Kane et al., 1979). Thus, simultaneous resistance to both RFM and TMP can be linked mechanistically.

To explore the development of multiple antibiotic resistance in opportunistic pathogens during long-term human habitation in space, an experiment involving the Biological Research in Canisters (BRIC) hardware on the ISS was devised. The mission, designated BRIC-18, is to be described elsewhere in detail. In preparation for the BRIC-18 mission, the present communication describes ground-based studies using spaceflight analogues: (i) a rotating wall vessel (RWV) clinostat served as a generator of simulated microgravity; (ii) two Gram-positive model organisms, Bacillus subtilis and Staphylococcus epidermidis, served as surrogates of opportunistic species found in human space habitats, and; (iii) RFM and TMP were selected as test antibiotics.

\section{MATERIALS AND METHODS}

\section{Bacterial Strains, Media, and Growth Conditions}

Strains used were B. subtilis WN1532 (trpC2) from the authors' strain collection and $S$. epidermidis strain ATCC12228 obtained from the American Type Culture Collection, Manassas, VA. Medium for general cultivation was Trypticase Soy Yeast Extract (TSY) medium containing (g/L): Tryptone, 15; Soytone, 5; NaCl, 5; Yeast Extract, 3; $\mathrm{K}_{2} \mathrm{HPO}_{4}$, 2.5; glucose, 2.5; final $\mathrm{pH}$ 7. For semisolid plates, agar was added to TSY at $15.0 \mathrm{~g} / \mathrm{L}$. As appropriate, the antibiotics RFM and TMP (Sigma-Aldrich) were added to TSY at final concentrations of $5 \mu \mathrm{g} / \mathrm{mL}$ and 5 $\mu \mathrm{g} / \mathrm{mL}$, respectively.

\section{Simulated Microgravity Experiments}

Simulated microgravity was provided using two 4-place Rotary Cell Culture Systems (RCCS-
4, Synthecon Inc., Houston, TX), each fitted with four 10-mL High Aspect Ratio Vessels (HARVs) (for details, refer to: http://www.synthecon.com/; accessed 11/6/14). In each experiment the two RCCS-4 units were operated simultaneously at 14 rpm, one unit in the vertical orientation producing simulated microgravity and the other in the horizontal orientation serving as the $1 \mathrm{~g}$ control. In accordance with the planned BRIC-18 flight experiment on ISS, cultures were incubated for 6 days at laboratory-ambient temperature $\left(\sim 23^{\circ} \mathrm{C}\right)$.

\section{Sample Analyses}

Cultures were transferred from HARV chambers into sterile $50-\mathrm{mL}$ conical centrifuge tubes. Optical densities at $660 \mathrm{~nm}\left(\mathrm{OD}_{660}\right)$ were determined in a spectrophotometer. For viable counts, cultures were diluted serially tenfold in Phosphate Buffered Saline (PBS), dilutions plated on TSY, and colonies counted after incubation at $37^{\circ} \mathrm{C}$ for 24 hours. To select for $\mathrm{RFM}^{\mathrm{R}}$ mutants, cultures were concentrated by centrifugation, plated without dilution onto TSY+RFM plates, and colonies counted after incubation at $37^{\circ} \mathrm{C}$ for 24 hours. The frequency of mutation to $\mathrm{RFM}^{\mathrm{R}}$ was calculated by dividing the total number of $R_{F} M^{R}$ mutants by the total number of viable cells in each culture. To determine the frequency of mutation to both RFM and TMP, single RFM ${ }^{R}$ colonies were picked onto TSY plates containing TMP and scored for growth after incubation at $37^{\circ} \mathrm{C}$ for 24 hours.

\section{Statistical Analyses}

Basic statistical parameters and One-Way Analysis of Variance (ANOVA) were computed using either Kaleidagraph version 3.6.2 (Synergy Software, Reading, PA), or an online statistical calculator (http://vassarstats.net/anova1u.html; accessed 11/6/14). Meta-analysis of data from multiple experiments was conducted using an online meta-analysis calculator (Health Decision Strategies, http://www.healthstrategy.com/meta/metainput.ht m; accessed 11/6/14).

\section{RESULTS}

Time Course of Bacillus $\begin{aligned} & \text { subtilis and } \\ & \text { Staphylococcus epidermidis } \\ & \text { Simulated Microgravity }\end{aligned}$
Growth in


As part of ground-based preparation for the BRIC-18 experiment to the ISS, B. subtilis and $S$. epidermidis cells were cultivated in the vertical (simulated microgravity) or horizontal $\left(\begin{array}{ll}1 & g\end{array}\right)$ orientation in clinostats. To match conditions under which cells were to be subjected on the ISS, cells were grown for 6 days at ISS ambient temperature $\left(\sim 23^{\circ} \mathrm{C}\right)$. To track the progress of growth, viable counts were determined from samples removed at daily intervals (Figure 1). $B$. subtilis cells initiated growth without a lag period and grew exponentially for the first 2 days at essentially the same rate, regardless of clinostat orientation (Figure 1A). In contrast, $S$. epidermidis cultures lagged for 1 day before entering exponential growth phase, and simulated microgravity-grown cultures appeared to grow at a slightly faster rate (Figure 1B). In both organisms, simulated microgravity-grown cultures grew to a slightly higher exponential cell number than did the $1 \mathrm{~g}$ controls (Figure 1). Once cultures reached the stationary phase, cell viability of both organisms was observed to decline; however, viability of cells cultured in simulated microgravity declined at a slower rate than in those grown at $1 \mathrm{~g}$ (Figure 1).
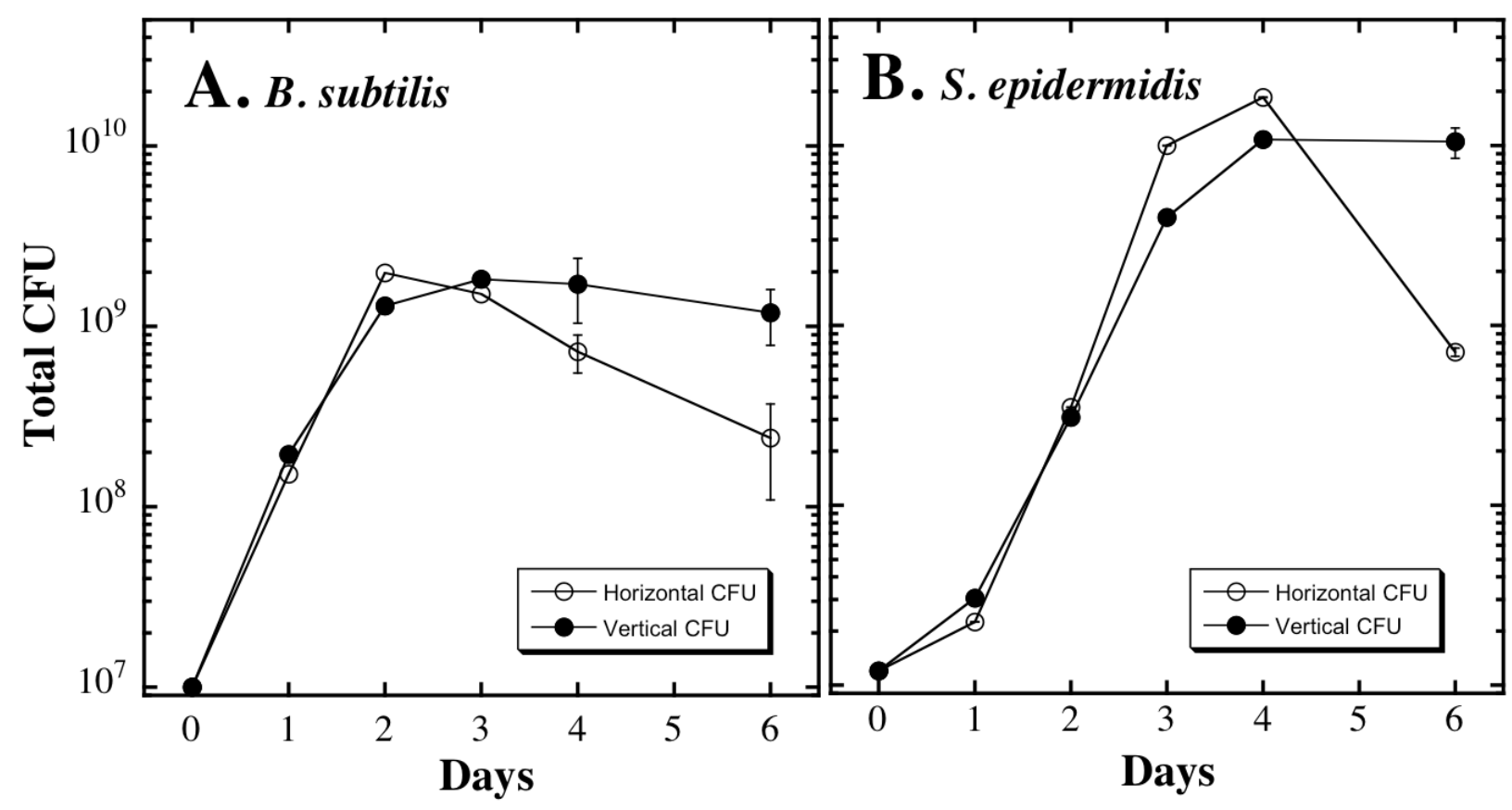

Figure 1. Time course of growth measured as viable counts of $B$. subtilis (A) and S. epidermidis (B) cells incubated in 10-mL HARVs either in simulated microgravity (vertical; filled circles) or $1 \mathrm{~g}$ (horizontal; open circles) orientation. Values are averages \pm standard deviations $(n=4)$.

\section{Six-Day Culture Experiments in Clinostats}

It was observed that both test organisms exhibited a higher final cell density after cultivation for 6 days in simulated microgravity compared to the $1 \mathrm{~g}$ controls (Figure 1). To more rigorously test this observation, both $B$. subtilis (Figure 2) and S. epidermidis (Figure 3) cells were cultivated for 6 days in 10-mL HARVs in simulated microgravity vs. $1 g(n=4)$. To achieve greater statistical power, multiple Trials (either 3 or 4) of each experiment were performed.

\section{Growth}

Six-day cultures of $B$. subtilis were harvested and growth was measured by optical density (OD) (Figure 2A) and viable counts (Figure 2B). In all three trials, $B$. subtilis cultures exposed to simulated microgravity exhibited greater cell mass, as measured by OD (Figure 2A), and 
greater numbers, as measured by viable counts (Figure 2B), than did cultures cultivated in parallel at $1 \mathrm{~g}$. Statistical analysis of the data by ANOVA revealed that in all 3 Trials the OD values were statistically significant (Figure 2A), and the viable count data was statistically significant in Trails 1 and 3, but not Trial 2 (Figure 2B).
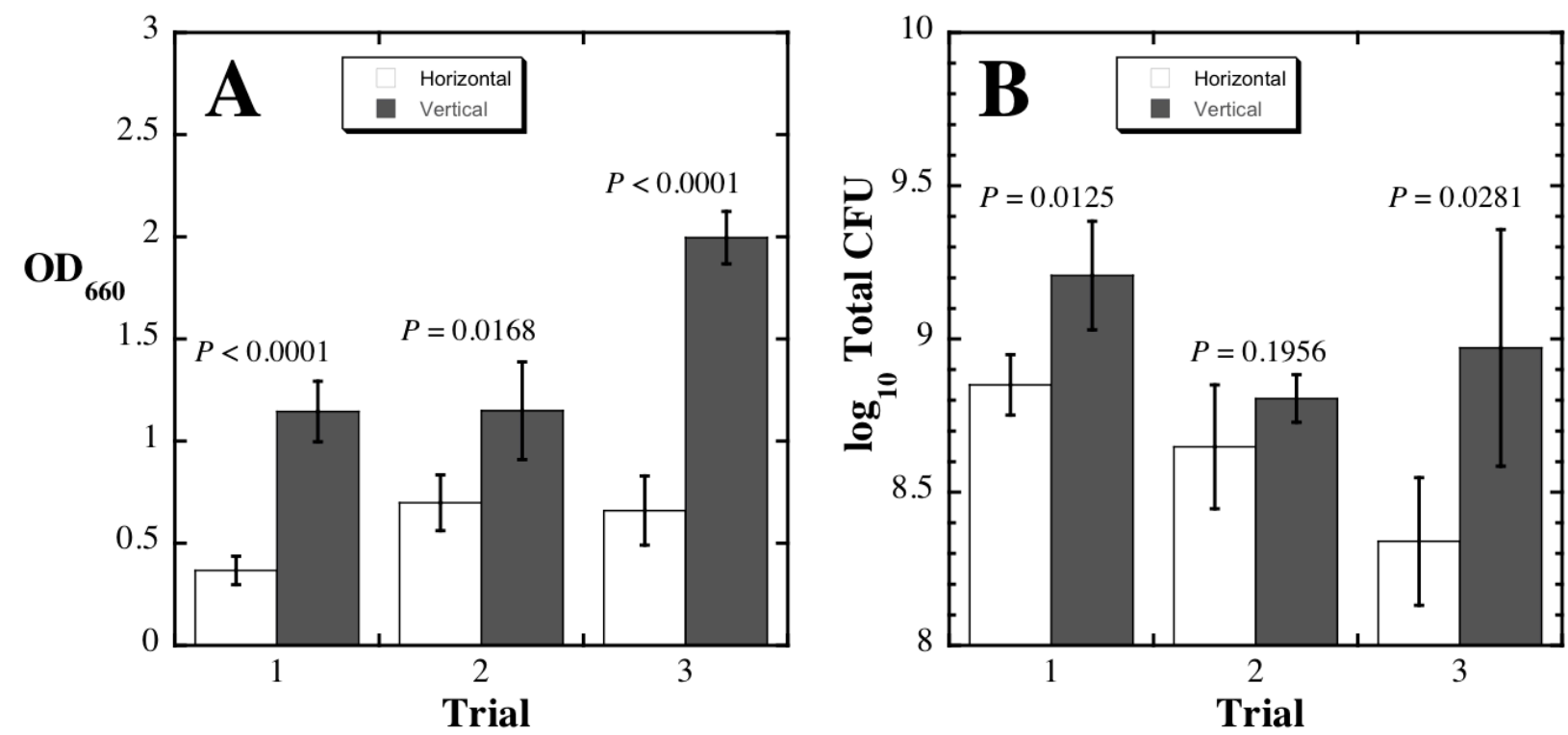

Figure 2. Optical density (A) and total cell number (B) of horizontal (open bars) and vertical (filled bars) cultures of $B$. subtilis cells after 6 days of incubation. Data are depicted as averages and standard deviations $(n=4)$ of three separate trials. Above each pair of bars is displayed the $P$ value derived from ANOVA. $P<$ 0.05 was considered statistically significant.

The same experiment was performed with cultures of S. epidermidis (Figure 3). Six-day cultures of $S$. epidermidis were harvested and growth was measured by OD (Figure 3A) and viable counts (Figure 3B). In all 3 Trials, simulated microgravity-grown cells exhibited significantly greater cells mass as measured by OD (Figure 3A). When viable counts were measured, it was observed that cell numbers were greater in simulated microgravity-grown cells in all 4 Trials, and the differences were statistically significant in Trials 2, 3, and 4, but not Trial 1 (Figure 3B).

\section{Frequency of mutation to $R F M^{R}$}

To determine the frequency of mutation to $\mathrm{RFM}^{\mathrm{R}}$, cells from the same cultures as described in Figures 2 and 3 were harvested, concentrated by centrifugation, and plated onto TSY plates containing RFM (Figure 4). When B. subtilis cultures were analyzed, a higher frequency of mutation to $\mathrm{RFM}^{\mathrm{R}}$ was observed in the simulated microgravity-grown cultures in all 3 Trials, and the differences were statistically significant by ANOVA in Trials 2 and 3 (Figure 4A). In sharp contrast, when $S$. epidermidis cultures were examined, the differences in mutation frequency in all 4 Trials were found to be not statistically significant, and indeed in Trials 2, 3, and 4 a lower frequency of mutation to RFM was noted in the simulated microgravity-grown cultures (Figure 4B). Thus it appeared mutation to $\mathrm{RFM}^{\mathrm{R}}$ was affected by simulated microgravity differently in B. subtilis vs. S. epidermidis.

Frequency of mutation to $R F M^{R}$ and $T M P^{R}$

$\mathrm{RFM}^{\mathrm{R}}$ resistant colony isolates were picked onto TSY containing TMP to score for $\mathrm{TMP}^{\mathrm{R}}$ 
(Figure 5). Data from B. subtilis showed that in all 3 Trials the simulated microgravity cultures exhibited a higher proportion of mutants resistant to both, RFM and TMP, than did the cultures from the $1 \mathrm{~g}$ controls; however, these differences were

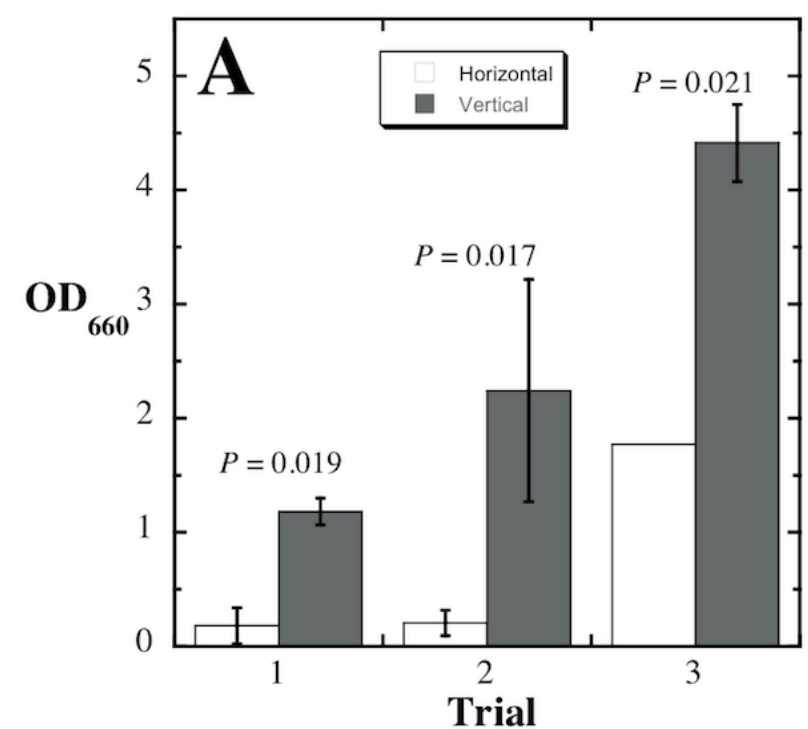

not significant at the $P<0.05$ level by ANOVA (Figure 5). In the case of $S$. epidermidis cultures, growth on TSY+TMP plates was slow and quite variable, leading to ambiguous results which are not reported here.

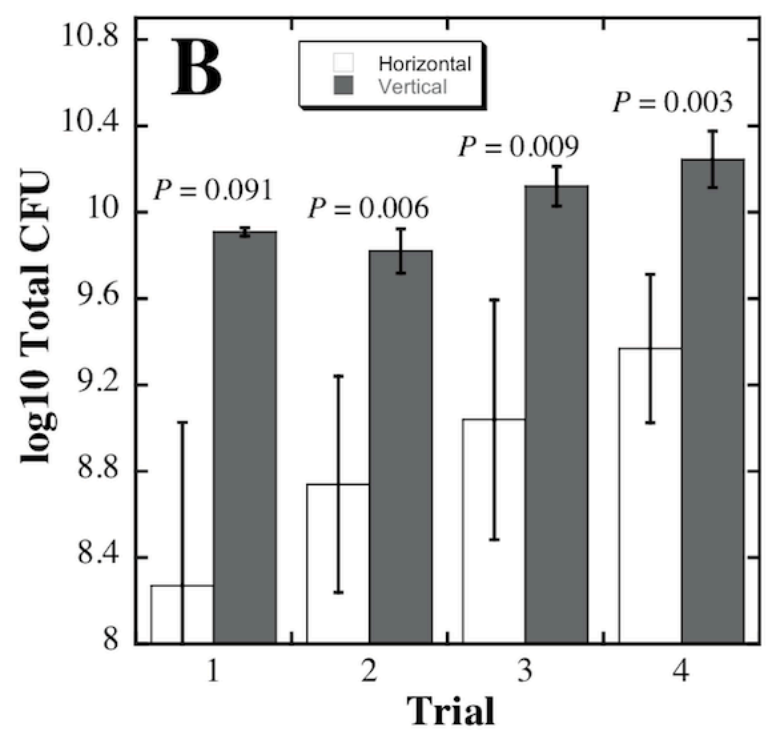

Figure 3. Optical density (A) and total cell number (B) of horizontal (open bars) and vertical (filled bars) cultures of $S$. epidermidis cells after 6 days of incubation. Data are depicted as averages and standard deviations $(n=4)$ of three (OD) or four (CFU) separate trials. Above each pair of bars is displayed the $P$ value derived from ANOVA. $P<0.05$ was considered statistically significant.
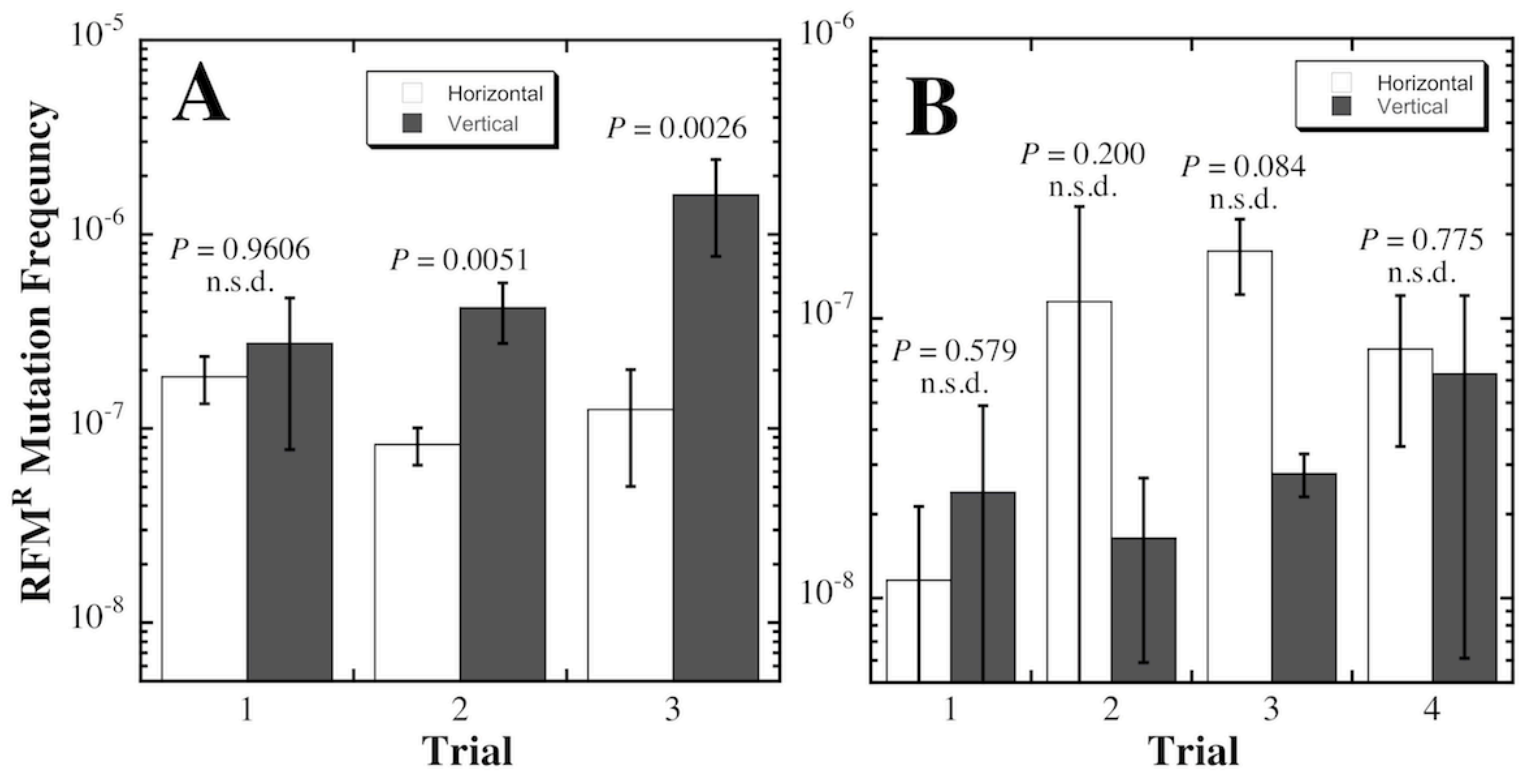

Figure 4. Mutation frequency to $\mathrm{RFM}^{\mathrm{R}}$ by $B$. subtilis 168 (A) and S. epidermidis (B) after 6 days of clinorotation in either the horizontal (open bars) or vertical (filled bars) orientation. Data are depicted as averages and standard deviations $(n=4)$ of three (A) or four (B) separate trials. Above each pair of bars is displayed the $P$ value derived from ANOVA. $P<0.05$ was considered statistically significant. 


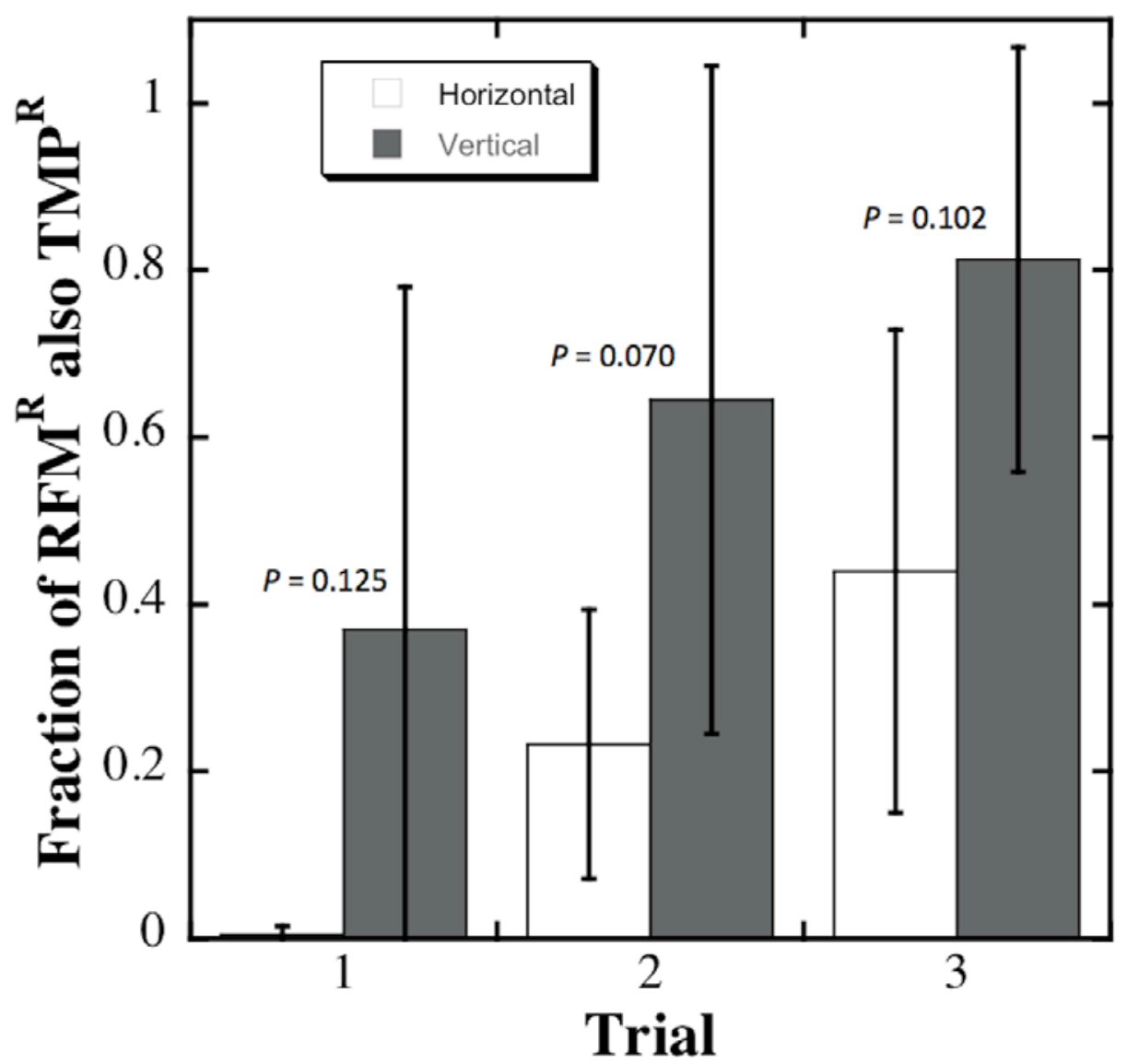

Figure 5. Frequency of simultaneous mutation to $R_{F} M^{R}$ and $T M P^{R}$ in $B$. subtilis. Data are averages and standard deviations $(n=4)$ from 3 independent trials.

\section{Meta-Analysis of the Data}

Meta-analysis is often used to increase statistical power for detection of small effects by analyzing and comparing data from multiple trials (Cohn and Becker, 2003). Therefore, metaanalysis was performed on the data collected in the present study. In the case of $B$. subtilis, metaanalysis revealed that all parameters measured (OD, viable counts, frequency of RFM ${ }^{\mathrm{R}}$ mutants, and frequency of $\mathrm{RFM}^{\mathrm{R}}$, TMP ${ }^{\mathrm{R}}$ double mutants), were significantly higher in cells cultivated in simulated microgravity (Figure 6). In contrast, meta-analysis revealed that in S. epidermidis, OD, and viable counts were significantly higher for cells grown in simulated microgravity, but that the frequency of mutation to $\mathrm{RFM}^{\mathrm{R}}$ was not (Figure 7).

\section{DISCUSSION}

Despite being a subject of intense research [reviewed in (Horneck et al., 2010; Klaus and Howard, 2006; Nickerson et al., 2004)], to date no coherent model has emerged adequately describing how microgravity affects bacterial growth and metabolism. This situation results from (i) infrequent opportunities to access spaceflight habitats, and (ii) limitations on the ability to perform sophisticated, well controlled on-board experiments in the spaceflight environment. Although it is impossible to replicate the microgravity environment on the surface of Earth, a number of ground-based systems have been designed to simulate the effects of microgravity (Anken, 2013). Among these, the RWV clinostat system has become 

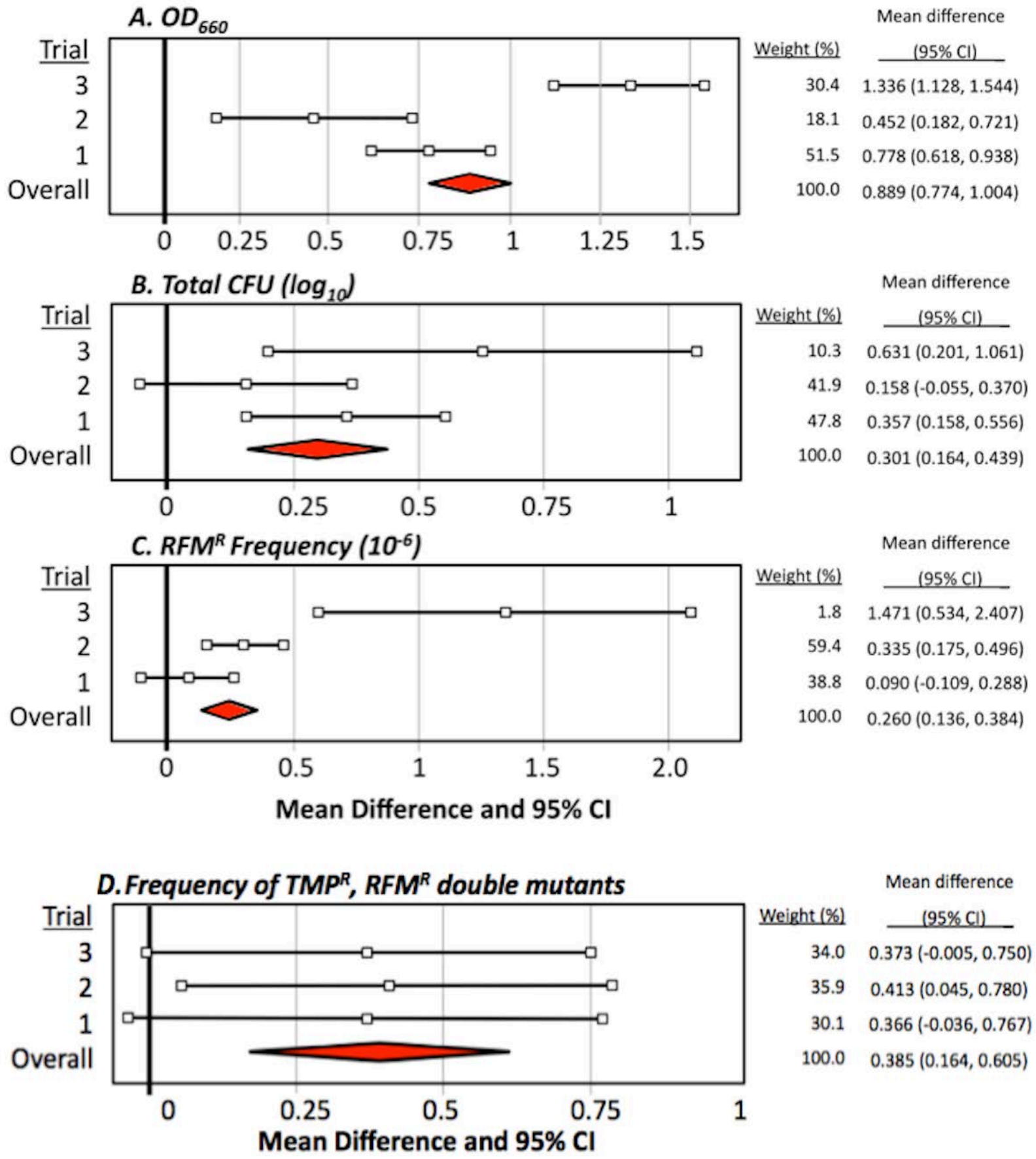

Figure 6. Meta-analysis of $B$. subtilis data for OD (A), Viable counts (B), frequency of mutation to $\operatorname{RFM}^{R}(C)$, and frequency of mutation to $\operatorname{RFM}^{R}$ and $T^{2} P^{R}(D)$. Relative weights, means, and $95 \%$ confidence intervals for each Trial are tabulated to the right and depicted graphically on the left. The red diamonds denote the overall means and $95 \%$ confidence intervals for the aggregate data. 

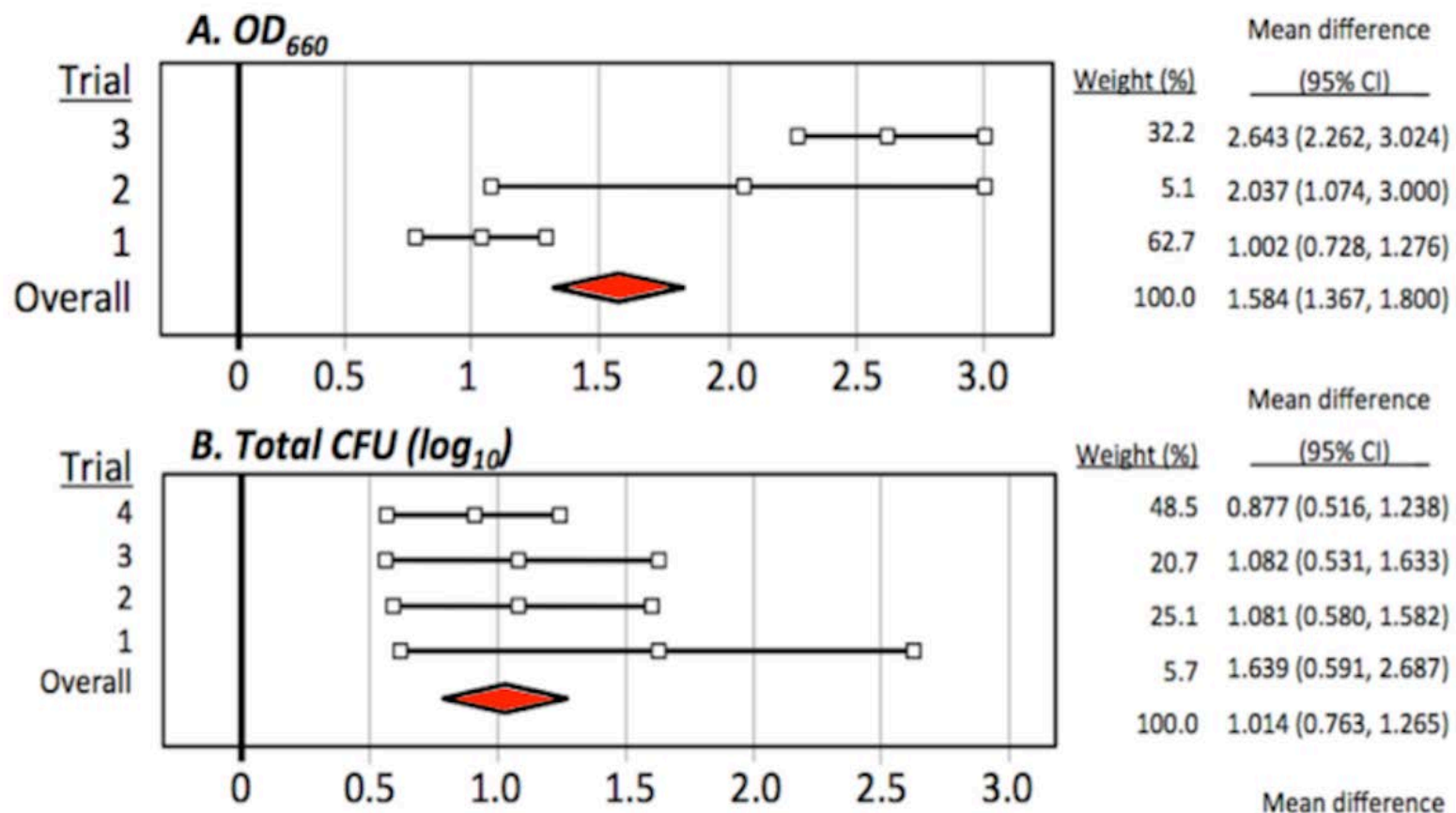

$20.71 .082(0.531,1.633)$

$25.11 .081(0.580,1.582)$

$5.71 .639(0.591,2.687)$

$100.01 .014(0.763,1.265)$

\section{RFM $^{R}$ Frequency $\left(10^{-7}\right)$}
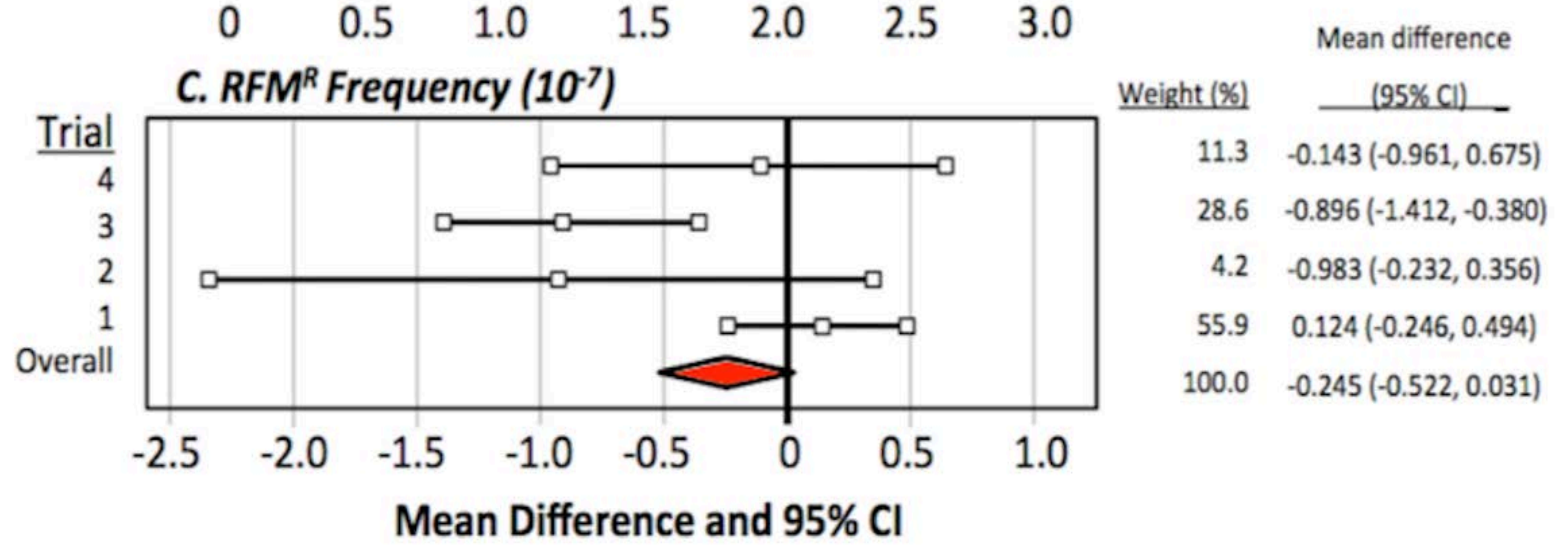

Figure 7. Meta-analysis of S. epidermidis data for OD (A), Viable counts (B), and frequency of mutation to $\operatorname{RFM}^{\mathrm{R}}(\mathrm{C})$. Relative weights, means, and $95 \%$ confidence intervals for each Trial are tabulated to the right and depicted graphically on the left. The red diamonds denote the overall means and $95 \%$ confidence intervals for the aggregate data.

widely used as a spaceflight culture analogue (Nickerson et al., 2003). In some cases, alterations in bacterial gene expression and virulence have been found to correlate well between clinostat and actual spaceflight experiments, but not in other cases [reviewed in (Rosenzweig et al., 2014)].

In this communication we report that $B$. subtilis and $S$. epidermidis cultures grew at essentially the same exponential rate and to similar cell densities in simulated microgravity and in $1 \mathrm{~g}$, in agreement with recent comparable experiments with clinostat-grown cultures of the Gram-negative bacteria Enterobacter cloacae, Escherichia coli, Citrobacter freudii, and Serratia marcsescens (Soni et al., 2014). It was observed that the number of viable cells declined during the stationary phase in both $B$. subtilis and $S$. epidermidis cultures, but that the rate of decline was markedly slower in simulated microgravitygrown cells of both species. In fact, the 
significantly higher OD values and numbers of viable cells in the simulated microgravity-grown cultures were not due to increased exponential growth of cells, but to decreased death of cells in the stationary phase. Because most natural environments are nutrient-limited, microbes in the environment likely spend much of their lives in a stationary phase-like condition (Chubukov and Sauer, 2014; Navarro Llorens et al., 2010). Thus, persistence in the stationary phase is an important mechanism for long-term survival of microorganisms in oligotrophic environments, such as those encountered within the ISS.

It was noted that simulated microgravitygrown $B$. subtilis cells demonstrated a significantly higher frequency of mutation to $\mathrm{RFM}^{\mathrm{R}}$ and to $\mathrm{RFM}^{\mathrm{R}} / \mathrm{TMP}^{\mathrm{R}}$ than did parallel $1 \mathrm{~g}$ grown cultures. As a possible reason for this observation, it has been established that both the model bacteria $B$. subtilis and $E$. coli possess wellcharacterized stationary-phase mutagenesis systems (Gonzalez et al., 2008; Robleto et al., 2012). Furthermore, induction of the stationaryphase mutagenesis system in $E$. coli by starvation has been shown to result in an elevation of clinically-relevant antibiotic resistance mutations (Petrosino et al., 2009). The results from the present experiments suggest that stress resulting from exposure to simulated microgravity in the RWV clinostat may be invoking a similar response in $B$. subtilis cells. In contrast, $S$. epidermidis cells did not exhibit a significant change in the frequency of mutation to $R_{F}{ }^{R}$ regardless of orientation in the clinostat. At present, the phenomenon of stationary-phase mutagenesis has not been well studied in Staphylococcus spp., but the results presented here suggest that if such a phenomenon exists, it appears to be insensitive to simulated microgravity supplied by clinorotation.

Previous studies of antibiotic resistance in microgravity have focused mainly on transient physiologic changes leading to increased or decreased antibiotic susceptibility (Klaus and Howard, 2006; Lapchine et al., 1986; Tixador et al., 1994; Tixador et al., 1985). In contrast, our results address the emergence of antibiotic resistance in microbes exposed to spaceflight stress resulting from mutations in genes encoding antibiotic targets. Investigation of both aspects is needed for a better understanding of the emergence of antibiotic resistance in the microbiome of the ISS, and the knowledge gained could be applied to similar confined settings on Earth.

The microgravity simulation experiments described here represent preliminary ground studies leading up to the BRIC-18 spaceflight mission to the ISS. Compilation of the ground simulation results reported here with upcoming actual spaceflight data will provide valuable insights into how these two microorganisms respond to the stresses of the integrated spaceflight environment, and possible implications for astronaut health.

\section{ACKNOWLEDGEMENTS}

This work was supported by a grant from the NASA Research Opportunities in Space Biology program (NNX12AN70G) to W.L.N. and P.F.-C., and by an internship from the NASA Florida Space Grant Consortium Training Grant (NNX10AM01H) to R.N. We also thank Ralf Moeller, Rafael Rodrigues de Oliveira, and Samantha Waters for their helpful advice and assistance.

\section{REFERENCES}

Anken R (2013) Simulation of microgravity for studies in gravitational biology: principles, devices, and applications. Current Biotechnology 2: 192-200

Campbell E, Korzheva N, Mustaev A, Murakami K, Nair S, Goldfarb A, Darst S (2001) Structural mechanism for rifampicin inhibition of bacterial RNA polymerase. Cell 104(6): 901-912

Centers for Disease Control and Prevention (CDC) (2011) Tuberculosis (TB): Treatment. Retrieved from http://www.cdc.gov/TB/ topic/treatment/default.htm (Accessed 11/7/14)

Chubukov V, Sauer U (2014) Environmental dependence of stationary-phase metabolism in Bacillus subtilis and Escherichia coli. Applied Environmental Microbiology 80: 2901-2909

Cohn LD, Becker BJ (2003) How meta-analysis increases statistical power. Psychological Methods 8: $243-253$ 
Cosgrove S, Avdic E (2013) Antibiotic Guidelines 2013-2014: Treatment Recommendations for Adult Inpatients. Baltimore, MD: The Johns Hopkins Antimicrobial Stewardship Program

Crucian B, Stowe RP, Ott CM, Becker JL, Haddon R, McMonigal KA, CF (2009) Risk of crew adverse health event due to altered immune response. In Human Research Program Human Health Coutermeasures Element, Vol. HRP-47060. Houston, TX: NASA Johnson Space Center

De Boever P, Mergeay M, Ilyin V, Forget-Hanus D, Van der Auwera G, Mahillon J (2007) Conjugation-mediated plasmid exchange between bacteria grown under spaceflight conditions. Microgravity Science and Technology 19: 138-144

Fukuda T, Fukuda K, Takahashi A, Ohnishi T, Nakano T, Sato M, Gunge N (2000) Analysis of deletion mutations of the rpsL gene in the yeast Saccharomyces cerevisiae detected after long-term flight on the Russian space station Mir. Mutation Research 470: 125-132

Gleckman R, Blagg N, Joubert DW (1981) Trimethoprim: mechanisms of action, antimicrobial activity, bacterial resistance, pharmacokinetics, adverse reactions, and therapeutic indications. Pharmacotherapy 1: 14-20

Gonzalez C, Hadany L, Ponder RG, Price M, Hastings PJ, Rosenberg SM (2008) Mutability and importance of a hypermutable cell subpopulation that produces stressinduced mutants in Escherichia coli. PLoS Genetics 4: e1000208

Guéguinou N, Huin-Schohn C, Bascove M, Bueb JL, Tschirhart E, Legrand-Frossi C, Frippiat JP (2009) Could spaceflight-associated immune system weakening preclude the expansion of human presence beyond Earth's orbit? Journal of Leukocyte Biology 86: 1027-1038

Horneck G, Klaus DM, Mancinelli RL (2010) Space microbiology. Microbiology and Molecular Biology Reviews 74: 121-156

Ilyin VK (2005) Microbiological status of cosmonauts during orbital spaceflights on Salyut and Mir orbital stations. Acta Astronautica 56: 839-850

International Space Exploration Coordination Group (ISECG) (2013) The Global
Exploration Roadmap. Washington, D.C.: NASA Headquarters

Jin DJ, Gross CA (1988) Mapping and sequencing of mutations in the Escherichia coli rpoB gene that lead to rifampicin resistance. Journal of Molecular Biology 202: 45-58

Kane JF, Wainscot VJ, Hurt MA (1979) Increased levels of dihydrofolate reductase in rifampinresistant mutants of Bacillus subtilis. Journal of Bacteriology 137: 1028-1030

Klaus DM, Howard HN (2006) Antibiotic efficacy and microbial virulence during spaceflight. Trends in Biotechnology 24: 131-136

Lapchine L, Moatti N, Gasset G, Richoilley G, Templier J, Tixador R (1986) Antibiotic activity in space. Drugs under Experimental and Clinical Research 12: 933-938

Maughan H, Callicotte V, Hancock A, Birky CW, Nicholson WL, Masel J (2006) The population genetics of phenotypic deterioration in experimental populations of Bacillus subtilis. Evolution 60: 686-695

Maughan H, Galeano B, Nicholson WL (2004) Novel rроB mutations conferring rifampin resistance on Bacillus subtilis: global effects on growth, competence, sporulation, and germination. Journal of Bacteriology 186: 2481-2486

National Research Council (NRC) (2014) Pathways to Exploration: Rationales and Approaches for a U.S. Program of Human Space Exploration. Washington, D.C.: National Academies Press

Navarro Llorens JM, Tormo A, Martínez-García E (2010) Stationary phase in gram-negative bacteria. Federation of European Microbiological Societies (FEMS) Microbiology Reviews 34: 476-495

Nicholson WL, Maughan H (2002) The spectrum of spontaneous rifampin resistance mutations in the $r p o B$ gene of Bacillus subtilis 168 spores differs from that of vegetative cells and resembles that of Mycobacterium tuberculosis. Journal of Bacteriology 184: 4936-4940

Nickerson CA, Ott CM, Wilson JW, Ramamurthy $\mathrm{R}$, LeBlanc CL, Höner zu Bentrup K, Hammond T, Pierson DL (2003) Low-shear modeled microgravity: a global environmental regulatory signal affecting 
bacterial gene expression, physiology, and pathogenesis. Journal of Microbiological Methods 54: 1-11

Nickerson CA, Ott CM, Wilson JW, Ramamurthy R, Pierson DL (2004) Microbial responses to microgravity and other low-shear environments. Microbiology and Molecular Biology Reviews 68: 345-361

Novikova ND (2004) Review of the knowledge of microbial contamination of the Russian manned spacecraft. Microbial Ecology 47: 127-132

Ott CM, Crabbé A, Wilson JW, Barrila J, Castro SL, Nickerson CA (2012) Microbial stress: spaceflight-induced alterations in microbial virulence and infectious disease risks for the crew. In Stress Challenges and Immunity in Space: From Mechanisms to Monitoring and Preventive Strategies, A Chouker (ed), pp 203-225. Berlin: Springer

Petrosino JF, Galhardo RS, Morales LD, Rosenberg SM (2009) Stress-induced betalactam antibiotic resistance mutation and sequences of stationary-phase mutations in the Escherichia coli chromosome. Journal of Bacteriology 191(19): 5881-5889

Robleto EA, Martin HA, Pedraza-Reyes M (2012) Mfd and transcriptional derepression cause genetic diversity in Bacillus subtilis. Frontiers in Bioscience 4: 1246-1254

Rosenzweig JA, Ahmed S, Eunson J, Chopra AK (2014) Low-shear force associated with modeled microgravity and spaceflight does not similarly impact the virulence of notable bacterial pathogens. Applied Microbiology and Biotechnology 98(21): 8797-8807

Sams CF (2009) The Human in Space: Lesson from ISS. In Proceedings of the 6th International Space Life Sciences Working Group. Sonanna, CA: NASA

Soni A, O'Sullivan L, Quick LN, Ott CM, Nickerson CA, Wilson JW (2014) Conservation of the low-shear modeled microgravity response in Enterobacteriaceae and analysis of the trp genes in this response. The Open Microbiology Journal 8: 51-58

Stein GE, Gurwith D, Gurwith M (1988) Randomized clinical trial of rifampintrimethoprim and sulfamethoxazoletrimethoprim in the treatment of localized urinary tract infections. Antimicrobial Agents and Chemotherapy 32: 802-806

Tixador R, Gasset G, Eche B, Moatti N, Lapchine L, Woldringh C, Toorop P, Moatti JP, Delmotte F, Tap G (1994) Behavior of bacteria and antibiotics under space conditions. Aviation, Space, and Environmental Medicine 65(6): 551-556

Tixador R, Richoilley G, Gasset G, Templier J, Bes JC, Moatti N, Lapchine L (1985) Study of minimal inhibitory concentration of antibiotics on bacteria cultivated in vitro in space (Cytos 2 experiment). Aviation, Space, and Environmental Medicine 56: 748-751

van Tongeren SP, Krooneman J, Raangs GC, Welling GW, Harmsen H (2007) Microbial detection and monitoring in advanced life support systems like the International Space Station. Microgravity Science and Technology 19(2): 45-48

Venkateswaran K, Vaishampayan P, Cisneros J, Pierson DL, Rogers SO, Perry J (2014) International Space Station environmental microbiome - microbial inventories of ISS filter debris. Applied Microbiology and Biotechnology 98(14): 6453-6466

Vickers AA, Potter NJ, Fishwick CW, Chopra I, O'Neill AJ (2009) Analysis of mutational resistance to trimethoprim in Staphylococcus aureus by genetic and structural modelling techniques. Journal of Antimicrobial Chemotherapy 63(6): 1112-1117

Wehrli W, Knüsel F, Schmid K, Staeheli M (1968) Interaction of rifamycin with bacterial RNA polymerase. Proceedings of the National Academy of Sciences USA 61(2): 667-673

Wotring VE (2012) Space Pharmacology, New York: Springer

Yatagai F, Saito T, Takahashi A, Fujie A, Nagaoka S, Sato M, Ohnishi T (2000) rpsL mutation induction after spaceflight on MIR. Mutation Research 453: 1-4

Zander J, Besier S, Faetke S, Saum SH, Müller V, Wichelhaus TA (2010) Antimicrobial activities of trimethoprim/sulfamethoxazole, 5-iodo-2'-deoxyuridine and rifampicin against Staphylococcus aureus. International Journal of Antimicrobial Agents 36(6): 562565 